\title{
Impact and Prediction of Lymph Node Involvement in Patients with Intrahepatic Cholangiocarcinoma After Curative Resection
}

\author{
NOBORU HARADA ${ }^{1}$, TOMOHARU YOSHIZUMI ${ }^{1}$, YO-ICHI YAMASHITA ${ }^{2}$, YUJI SOEJIMA ${ }^{1}$, \\ TORU IKEGAMI ${ }^{1}$, NORIFUMI HARIMOTO $^{1}$, SHINJI ITOH $^{1}$ and YOSHIHIKO MAEHARA ${ }^{1}$ \\ ${ }^{1}$ Department of Surgery and Medical Science, Graduate School of Medical Sciences, \\ Kyushu University, Fukuoka, Japan; \\ ${ }^{2}$ Department of Gastroenterological Surgery, Graduate School of Life Science, \\ Kumamoto University, Kumamoto, Japan
}

\begin{abstract}
Background: The aim of this study was to identify the preoperative predictors of prognosis in patients with intrahepatic cholangiocarcinoma (ICC) undergoing resection. Patients and Methods: We enrolled 90 patients with ICC who underwent surgical resection, including 59 in whom surgery was considered curative, and measured the overall survival $(O S)$, recurrence-free survival (RFS), and other outcomes and potential prognostic factors. Results: Multivariate Cox proportional hazards analysis showed that tumor in the resection margins $(R>0)$ independently predicted long-term $O S$ in the whole cohort. In the curatively-resected group (RO), lymph node involvement was the only independent predictor of long-term OS. Multiple tumors, perihilar tumor location and serum carcinoembryonic antigen (CEA) concentration $>2.2 \mathrm{ng} / \mathrm{ml}$ were independent predictors of lymph node involvement before curative resection. Conclusion: Patients with ICC with multiple tumors, perihilar tumors and serum CEA concentration $>2.2 \mathrm{ng} / \mathrm{ml}$ in association with lymph node involvement may need additional preoperative chemotherapy.
\end{abstract}

Intrahepatic cholangiocarcinoma (ICC) is a primary liver cancer arising in the intrahepatic bile ducts. It is the second most common primary hepatic tumor after hepatocellular carcinoma, comprising about $3.3 \%$ of all hepatic tumors in Japan (1). The global incidence of ICC is reportedly rising (2).

To date, surgical resection remains the only potentially curative treatment for ICC (3), but early recurrence is common

Correspondence to: Noboru Harada, MD, Ph.D., Department of Surgery and Medical Science, Graduate School of Medical Sciences, Kyushu University, 3-1-1 Maidashi, Higashi-ku, Fukuoka, 812-8582, Japan. Tel: +81 926425466, Fax: +81 926425482, e-mail: nharada@surg2.med.kyushu-u.ac.jp

Key Words: Lymph node metastasis, R0 resection, perihilar. even in patients who have undergone curative resection (R0), with 5-year overall survival (OS) rates after curative resection reported to be $58 \%$ (4). Nevertheless, a surgical approach is the only means by which a cure can be achieved. It is necessary to improve our understanding of the factors that influence outcome after $\mathrm{R} 0$ resection for ICC, as there are no widely accepted guidelines to inform postoperative treatment strategy. Moreover, a better understanding of the preoperative factors associated with poor prognosis in patients with ICC scheduled for curative resection would inform decisions about the need for additional preoperative treatment in high-risk patients, such as neoadjuvant chemotherapy. The evidence supporting the therapeutic benefits of neo- and adjuvant treatment relies on retrospective series or relatively small prospective studies (5-7). It is essential to be able to identify patients at risk of recurrence despite complete curative resection of ICC so that neo- and adjuvant chemotherapy can be more effectively targeted and outcomes can be improved.

The aim of this study was to examine the outcomes of surgery in patients with ICC, and to identify the preoperative predictors of OS in those undergoing curative resection.

\section{Patients and Methods}

Study design. We enrolled 90 patients with Union for International Cancer Control (UICC) stages I-IV ICC (8) who had undergone surgical resection with curative intent at the Department of Surgery and Science, Graduate School of Medical Sciences, Kyushu University, Fukuoka, Japan, between April 1986 and August 2016. All patients had a confirmed pathological diagnosis of a malignancy arising from the intrahepatic bile ducts. Patients' demographic and clinical characteristics, including perioperative factors, tumor characteristics and patient survival were analyzed, and factors predicting OS and disease-free survival (DFS) were evaluated by univariate and multivariate analyses. Written informed consent was obtained from all patients for surgical treatment according to institutional guidelines. The study protocol conformed to the ethical guidelines of the 1975 Helsinki Declaration and was approved by our Institutional Review Board (approval number: 29-72). 
Surgical treatment. All patients underwent dynamic computed tomographic imaging preoperatively. We classified ICC tumors as perihilar or peripheral, as previously described (9). The details of our surgical techniques and patient follow-up methods have been reported previously $(4,10,11)$. Major hepatectomy with bile duct resection was performed when bile duct invasion was suspected to have affected the first hepatic duct. Partial hepatectomy was performed for peripheral ICC without bile duct invasion. When we considered it desirable to confirm the surgical margins, we sent the resected specimen for frozen pathology (4). The right and left lobes of the liver have different routes of lymphatic drainage; therefore, the technique for lymph node dissection was tailored to the location of the primary tumor (10). Postoperative surgical complications were recorded according to the Clavien-Dindo classification (12).

Histopathological examination. After tumor resection, all specimens were subject to histopathologic examination and classified based on the predominant pathologic findings as well-, moderately, or poorly differentiated tubular adenocarcinoma. Surgical margins were regarded as positive $(\mathrm{R}>0)$ if infiltrating adenocarcinoma was present at the hepatic transection line, the proximal or distal bile duct transection line or the dissected periductal soft tissue margins. The final stage of each intrahepatic cholangiocarcinoma was determined pathologically according to the UICC TNM classification system, seventh edition (8).

Statistical analysis. For continuous variables, non-parametric analyses were performed using Wilcoxon rank-sum tests. Categorical variables were compared using chi-squared or Fisher's exact tests. Cut-off values for continuous variables were calculated as the median values of the whole study population. Survival curves were constructed using the Kaplan-Meier method and compared by univariate log-rank (Mantel-Cox) tests. Factors found to be significant on univariate analysis were subjected to multivariate analysis using a Cox proportional hazards model. To identify factors independently predictive of pathologic lymph node involvement, factors found to have a $p$-value of less than 0.02 on univariate analysis were assessed by multivariate logistic regression analyses. Otherwise, a $p$-value of less than 0.05 was considered statistically significant. Statistical analyses were performed using JMP software (version 12.2.0; SAS Institute, Cary, NC, USA).

\section{Results}

Patient and tumor characteristics, and surgical treatment. The 90 patients included 55 men and 35 women, of median age 61 years (range $=33-82$ years). All tumors were tubular adenocarcinomas, $79(87.8 \%)$ were classified as being moderately or poorly differentiated. Lymph node metastasis was present in 27 cases $(30.0 \%)$. Curative (R0) resections were performed in 59 patients $(65.6 \%)$. Patient demographic and tumor characteristics are summarized in Table I.

Comparison of surgical outcomes in whole cohort. The median duration of follow-up after surgery for the entire cohort of 90 patients was 20.4 months (range=0.4-292 months). At the time of writing, 46 patients $(51.1 \%)$ had died. The 1-, 3-, and 5-year OS rates for the whole cohort of
Table I. Patient demographics, tumor characteristics, and surgical outcomes in patients with intrahepatic cholangiocarcinoma $(n=90)$.

\begin{tabular}{lc}
\hline Characteristic & Value \\
\hline Patient demographics & \\
Median age (range), years & $61(33-82)$ \\
Male:female, $\mathrm{n}$ & $55: 35$ \\
Hepatitis B infection: Yes:no, $\mathrm{n}$ & $11: 79$ \\
Hepatitis C infection: Yes:no, $\mathrm{n}$ & $13: 77$ \\
Median ICG R15 (range), \% & $10(0-32.7)$ \\
Child-Pugh class: A:B, $\mathrm{n}$ & $70: 20$ \\
Median CEA level (range), ng/ml & $2.2(0.2-117.5)$ \\
Median CA19-9 level (range), mAU/l & $38(0-680000)$ \\
Tumor characteristics & \\
Median tumor size (range), cm & $4.2(1-12)$ \\
Well:mod:poor, $\mathrm{n}$ & $11: 36: 43$ \\
Microvascular invasion: Yes:no, $\mathrm{n}$ & $37: 53$ \\
Microvascular invasion: Yes:no, $\mathrm{n}$ & $30: 60$ \\
Lymphatic invasion: Yes:no, $\mathrm{n}$ & $34: 56$ \\
Lymph node invasion: Yes:no, $\mathrm{n}$ & $27: 63$ \\
Intrahepatic metastasis: Yes:no, $\mathrm{n}$ & $38: 52$ \\
UICC stage I:II:III:IVA:IVB, $\mathrm{n}$ & $31: 13: 14: 25: 7$ \\
Surgical outcomes & \\
Hepatectomy: Major:minor, $\mathrm{n}$ & $59: 31$ \\
Median surgical time (range), min & $363(142-943)$ \\
Median surgical blood loss (range), g & $900(0-15510)$ \\
Transfusion: Yes:no, $\mathrm{n}$ & $30: 60$ \\
R0:R1:R2, $\mathrm{n}$ & $59: 19: 12$ \\
Median hospital stay (range), days & $21(7-131)$ \\
Postoperative complication*: Yes:no, $\mathrm{n}$ & $38: 52$ \\
\hline
\end{tabular}

ICGR15: Indocyanine green retention rate at 15 minutes; Well:mod:poor: well-:moderately:poorly differentiated; CEA: carcinoembryonic antigen; CA19-9: carbohydrate antigen 19-9; UICC: Union for International Cancer Control. Major hepatectomy indicates lobectomy or greater. *Clavien-Dindo grade $\geq$ II.

90 patients were $74.4 \%, 54.1 \%$ and $45.9 \%$, respectively (Figure 1A), and the cor responding DFS rates were $55.5 \%$, $40.5 \%$ and $36.3 \%$, respectively (Figure 1B).

Clinicopathological factors were evaluated to determine useful prognostic factors in this population (Table II). Univariate analysis showed that tumor size $>4 \mathrm{~cm}(p=0.01)$, multiple tumors $(p<0.0001)$, poorly differentiated adenocarcinoma $(p=0.0008)$, microvascular venous invasion $(p<0.0001)$, lymph node involvement $(p<0.0001)$, intrahepatic metastasis $(p<0.0001)$, tumor expansive growth $(p=0.03)$, lymphatic invasion $(p<0.0001)$, surgical blood loss $>900 \mathrm{ml}(p=0.01)$, intraoperative transfusion $(p=0.005)$ and $\mathrm{R}>0$ ( $p=0.0003)$ were significantly associated with poorer long-term survival. Multivariate Cox proportional hazards analysis showed that poorly differentiated adenocarcinoma [hazard ratio $(\mathrm{HR})=2.5,95 \%$ confidence interval $(\mathrm{CI})=1.3$ $5.0 ; p=0.005]$ and $\mathrm{R}>0(\mathrm{HR}=2.7,95 \% \mathrm{CI}=1.2-6.0, p=0.010)$ were independent predictors of poorer long-term OS. 


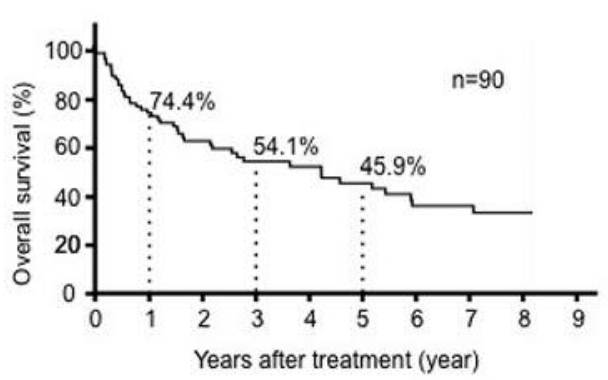

B

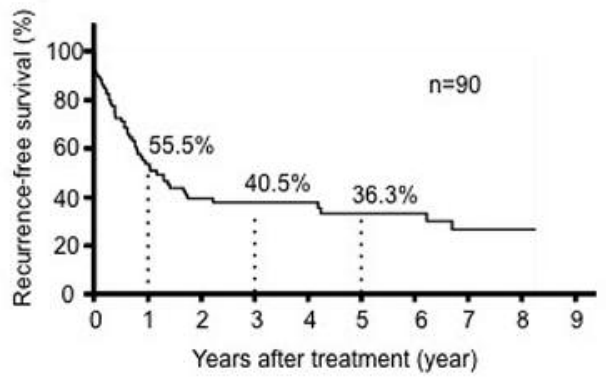

Figure 1. Cumulative overall (OS; A) and disease-free; B) survival curves for the whole cohort of patients with intrahepatic cholangiocarcinoma $(n=90)$ after surgical resection (Kaplan-Meier method).

Comparison of surgical outcomes in the $R 0$ resection group. The 1-, 3-, and 5-year OS rates for the R0 resection group $(\mathrm{n}=59)$ were $78.9 \%, 68.2 \%$ and $57.9 \%$, respectively, while the 1 - and 3-year OS rates for the $\mathrm{R}>0$ resection group $(\mathrm{n}=31)$ were $65.8 \%$ and $21.6 \%$, respectively $(p=0.0003$; Figure 2). Clinicopathological factors were also evaluated to determine prognostic factors in this population (Table III). Univariate analysis showed that multiple tumors $(p=0.01)$, microvascular venous invasion $(p=0.0003)$, lymph node involvement $(p<0.0001)$, intrahepatic metastasis $(p<0.0001)$, lymphatic invasion $(p=0.002)$, surgical blood loss $>900 \mathrm{ml}$ $(p=0.01)$ and intraoperative transfusion $(p=0.008)$ were significantly associated with poorer long-term survival. Multivariate Cox proportional hazards analysis showed that lymph node involvement $(\mathrm{HR}=4.3,95 \% \mathrm{CI}=1.4-15.6$; $p=0.010$ ) was the only independent predictor of poorer longterm OS in the R0 group.

Comparison of surgical outcomes after $R O$ resection in patients with and without lymph node involvement. As shown in Table IV, univariate analysis identified four variables as risk factors for lymph node involvement in patients with ICC undergoing R0 resection: tumor location in the left lobe $(p=0.017)$, perihilar tumor location $(p=0.04)$, multiple tumors $(p=0.02)$ and serum CEA concentration $>2.2 \mathrm{ng} / \mathrm{ml}(p=0.11)$. Multivariate logistic regression analysis identified multiple tumors $(\mathrm{HR}=4.7,95 \% \mathrm{CI}=1.07-23.4 ; p=0.041)$, perihilar tumor location $(\mathrm{HR}=7.0,95 \% \mathrm{CI}=1.01-147 ; p=0.049)$ and CEA concentration $>2.2 \mathrm{ng} / \mathrm{ml}(\mathrm{HR}=4.3,95 \% \mathrm{CI}=1.00-20.2$; $p=0.0496)$ as independent preoperative predictors of lymph node involvement before $\mathrm{R} 0$ resection (Table $\mathrm{V}$ ).

Comparison of surgical outcomes in patients with RON1 and $R>0$ tumors. The OS rate in R0 patients with lymph node involvement (R0N1) was comparable to that of those with $\mathrm{R} 2$ disease $(p=0.482)$, but the OS rate in the R0N1 resection group was significantly worse than that of those in the R1

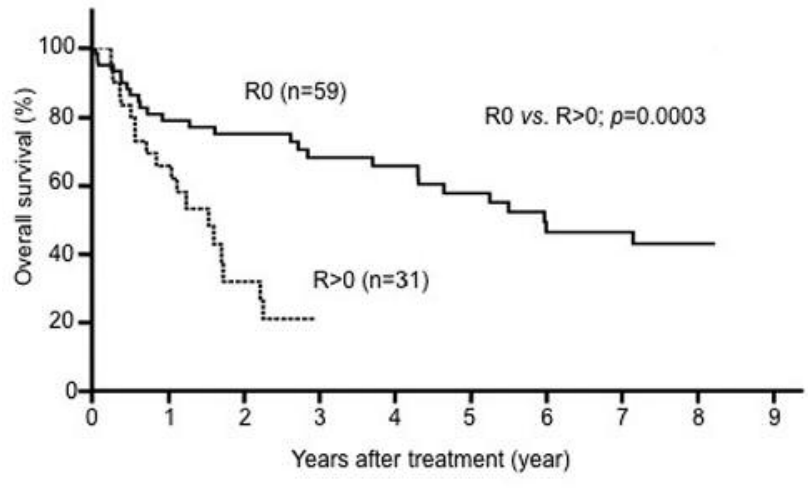

Figure 2. Cumulative overall survival (OS) curves for patients in the RO resection group and $R>0$ group. The 1-, 3-, and 5-year OS rates for the $R 0$ resection group were $78.9 \%, 68.2 \%$, and $57.9 \%$, respectively, whereas the 1 - and 3-year rates for the $R>0$ resection group were $65.8 \%$ and $21.6 \%$, respectively $(p=0.0003)$.

resection group ( $p=0.049$; Figure $3 \mathrm{~A}$ ). The recurrence-free survival (RFS) rate in the R0N1 group was comparable to that of the R1-resection group ( $p=0.24)$, while that for the R0N0 resection group was significantly better than those for the R1 and R0N1 groups $(p=0.0003$ and $p<0.0001$, respectively; Figure 3B). The survival rate of patients with recurrent R0N1 disease $(0 \%)$ was significantly worse than that of those with $\mathrm{R} 1$ disease $(36 \%, p=0.040)$.

The influence of lymph node involvement after R0 resection is summarized in Table VI. The RFS rate for the R0N1 resection group was significantly inferior to that of the R0N0 group $(p<0.0001$; Figure $3 \mathrm{~B})$. The peritoneal dissemination rate in the lymph node-positive group was greater than that of the lymph node-negative group $(p=0.002)$, while the single liver metastasis rate of the lymph node-negative group was greater than that of the lymph node-positive group ( $p=0.020$; Table IV). 
Table II. Univariate and multivariate overall survival analyses of prognostic factors in surgical patients with intrahepatic cholangiocarcinoma $(n=90)$.

\begin{tabular}{|c|c|c|c|c|}
\hline \multirow[b]{2}{*}{ Factor } & \multicolumn{2}{|c|}{ Univariate } & \multicolumn{2}{|c|}{ Multivariate } \\
\hline & 5 -Year survival rate $(\%)$ & $p$-Value & Hazard ratio $(95 \% \mathrm{CI})$ & $p$-Value \\
\hline Age: $>61 / \leq 61$ years & $49.7 / 44.5$ & 0.96 & & \\
\hline Tumor size: $>4 / \leq 4 \mathrm{~cm}$ & $29.7 / 65.7$ & 0.01 & $1.3(0.7-2.5)$ & 0.46 \\
\hline Multiple tumors: Yes/no & $18.6 / 56.7$ & $<0.0001$ & $1.0(0.4-2.6)$ & 0.96 \\
\hline Histological grading: Poor/well- or moderate & $23.6 / 67.6$ & 0.0008 & $2.5(1.3-5.0)$ & 0.005 \\
\hline Microvascular invasion: Yes/no & $34.6 / 54.1$ & 0.38 & & \\
\hline Microvascular invasion: Yes/no & $16.9 / 61.7$ & $<0.0001$ & $1.7(0.7-4.2)$ & 0.21 \\
\hline Lymphnode involvement: Yes/no & $6.4 / 60.9$ & $<0.0001$ & $2.0(0.8-4.9)$ & 0.12 \\
\hline Intrahepatic metastasis: Yes/no & $18.0 / 66.6$ & $<0.0001$ & $2.1(0.8-5.4)$ & 0.15 \\
\hline Expansive growth: Yes/no & $33.5 / 57.7$ & 0.03 & $1.5(0.7-3.3)$ & 0.35 \\
\hline Lymphatic invasion: Yes/no & $28.0 / 56.9$ & $<0.0001$ & $1.9(0.7-4.8)$ & 0.18 \\
\hline CEA level: $>2.2 / \leq 2.2 \mathrm{ng} / \mathrm{ml}$ & $48.8 / 45.2$ & 0.97 & & \\
\hline CA19-9 level: $>38 / \leq 38 \mathrm{mAU} / 1$ & $53.9 / 39.7$ & 0.20 & & \\
\hline Surgical time: $>363 / \leq 363 \mathrm{~min}$ & $49.2 / 42.5$ & 0.19 & & \\
\hline Surgical blood loss: $>900 / \leq 900 \mathrm{~g}$ & $63.4 / 34.8$ & 0.01 & $1.1(0.5-2.7)$ & 0.81 \\
\hline Intraoperative transfusion: Yes:no & $31.1 / 54.3$ & 0.005 & $2.1(0.98-5.0)$ & 0.06 \\
\hline $\mathrm{R}>0$ vs. $\mathrm{R} 0$ & $57.9 / 9.2$ & 0.0003 & $2.7(1.2-6.0)$ & 0.01 \\
\hline
\end{tabular}

CI: Confidence interval, CEA: carcinoembryonic antigen; CA19-9: carbohydrate antigen 19-9.

Table III. Univariate and multivariate overall survival analyses of prognostic factors in patients with ICC after RO resection.

\begin{tabular}{|c|c|c|c|c|}
\hline \multirow[b]{2}{*}{ Factor } & \multicolumn{2}{|c|}{ Univariate } & \multicolumn{2}{|c|}{ Multivariate } \\
\hline & 5 -Year survival rate $(\%)$ & $p$-Value & Hazard ratio $(95 \% \mathrm{CI})$ & $p$-Value \\
\hline Age: $>61 / \leq 61$ years & $56.9 / 61.5$ & 0.75 & & \\
\hline Tumor size: $>4 / \leq 4 \mathrm{~cm}$ & $72.6 / 44.2$ & 0.1 & & \\
\hline Multiple tumors: Yes/no & $30.8 / 67.1$ & 0.01 & $0.7(0.2-2.2)$ & 0.49 \\
\hline Histological grading: Poor/well- or moderate & $69.9 / 39.9$ & 0.19 & & \\
\hline Microvascular invasion: Yes/no & $45.8 / 65.3$ & 0.78 & & \\
\hline Microvascular invasion: Yes/no & $26.5 / 73.7$ & 0.0003 & $1.6(0.4-5.6)$ & 0.47 \\
\hline Lymphnode involvement: Yes/no & $11.5 / 71.6$ & $<0.0001$ & $4.3(1.4-15.6)$ & 0.01 \\
\hline Intrahepatic metastasis: Yes/no & $22.0 / 78.8$ & $<0.0001$ & $3.2(0.7-14.5)$ & 0.13 \\
\hline Expansive growth: Yes/no & $48.0 / 73.3$ & 0.07 & & \\
\hline Lymphatic invasion: Yes/no & $38.7 / 69.0$ & 0.002 & $1.5(0.5-4.7)$ & 0.47 \\
\hline CEA level: $>2.2 / \leq 2.2 \mathrm{ng} / \mathrm{ml}$ & $59.0 / 57.5$ & 0.62 & & \\
\hline CA19-9 level: $>38 / \leq 38 \mathrm{mAU} / 1$ & $71.2 / 42.7$ & 0.054 & & \\
\hline Surgical time: $>363 / \leq 363 \mathrm{~min}$ & $69.9 / 49.5$ & 0.11 & & \\
\hline Surgical blood loss: $>900 / \leq 900 \mathrm{~g}$ & $76.8 / 47.0$ & 0.01 & $3.2(0.83-12.7)$ & 0.09 \\
\hline Intraoperative transfusion: Yes:no & $40.7 / 68.2$ & 0.008 & $1.4(0.5-4.3)$ & 0.56 \\
\hline
\end{tabular}

CI: Confidence interval, Well:mod:poor: well-:moderately:poorly differentiated; CEA: carcinoembryonic antigen; CA19-9: carbohydrate antigen 19-9.

\section{Discussion}

The prognosis of patients with ICC remains unsatisfactory even with curative resection, due to the high recurrence rate. The OS and DFS rates of our cohort were comparable with those reported previously $(4,12,13)$. For the whole cohort, $\mathrm{R} 0$ resection was an independent predictor of long-term OS, unless the tumor was poorly differentiated. We also examined prognostic factors in the R0 group as their 5-year OS was unsatisfactory despite curative resection (57.9\%). 
A

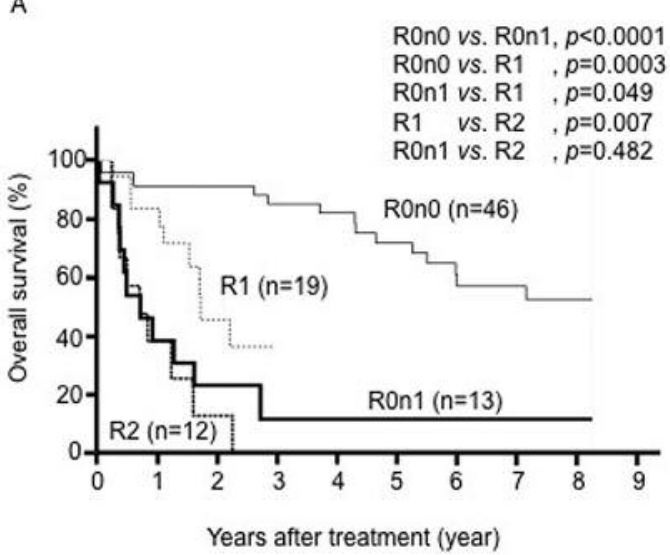

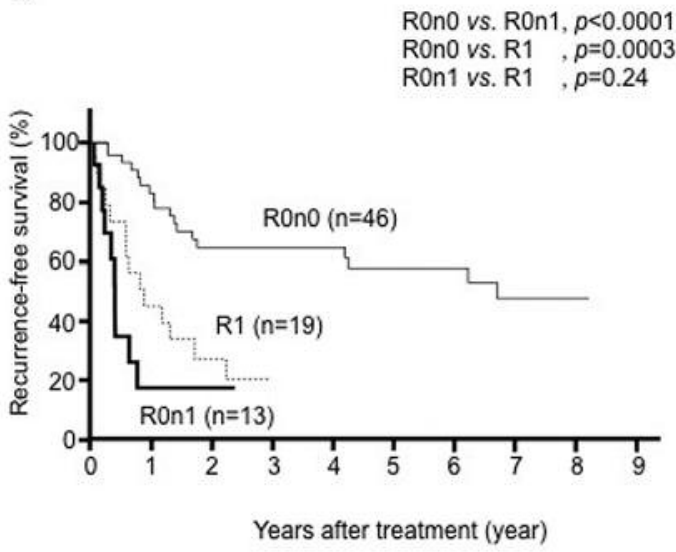

Figure 3. Cumulative overall survival (OS; A) and recurrent-free (RFS; B) survival curves for patients with intrahepatic cholangiocarcinoma according to resection status. The OS rate in the R0n1 resection group was comparable to that for the R2 resection group ( $p=0.482)$, but was significantly worse than that for the R1 resection group $(p=0.049)$. The RFS rate for the R0n1 resection group was comparable to that for the R1 resection group $(p=0.24)$, while that for the R0n0 resection group was significantly better than those for the $R 1$ resection and R0n1 groups ( $p=0.0003$ and $p<0.0001$, respectively).

Table IV. Comparison of surgical outcomes after RO resection for intrahepatic cholangiocarcinoma in patients with and without lymph node (LN) involvement.

\begin{tabular}{llccc}
\hline Factor & Subgroup & With LN involvement (n=46), & Without LN involvement (n=13), $\mathrm{n}$ & $p$-Value \\
\hline Patient demographics & Age>61 years & 19 & 7 & 0.42 \\
& Male:female & $26: 20$ & 2 & 0.22 \\
& Hepatitis B infection & 6 & 1 & 0.85 \\
& Hepatitis C infection & 8 & $11: 2$ & 0.35 \\
& Child-Pugh class: A:B & $36: 10$ & 8 & 0.62 \\
Tumor characteristics & Tumor size>4 cm & 21 & $9: 4$ & 0.31 \\
& Tumor location: Left:right & $22: 24$ & $72: 1$ & 0.17 \\
& Perihilar:peripheral & $31: 15$ & 8 & 0.04 \\
& Multiple tumors & 7 & 0.02 \\
& CEA level>2.2 ng/ml & 16 & 0.11 \\
& CA19-9 level>38 mAU/l & 18 & $6: 7$ & 0.21 \\
\hline
\end{tabular}

Well:mod:poor: Well-:moderately:poorly differentiated; CEA: carcinoembryonic antigen; CA19-9: carbohydrate antigen 19-9.

We found that lymph node metastasis was the only factor that predicted survival in the R0-resection group. Surgery alone may, therefore, be insufficient, even in patients who undergo curative resection, due to the presence of lymph node metastasis.

Lymph node metastasis is reportedly to be the most significant predictor of poor outcome in ICC, but extended lymphadenectomy does not improve survival $(4,10,14)$. Lymphatic invasion is the major mode of metastasis in ICC (14). Although most hepatic lymph flows into the hilar nodes
Table V. Multivariate analysis of preoperative risk factors for lymph node involvement in patients with intrahepatic cholangiocarcinoma.

\begin{tabular}{lccc}
\hline Variable & Odds ratio & $\begin{array}{c}95 \% \text { Confidence } \\
\text { interval }\end{array}$ & $p$-Value \\
\hline Tumor location: Left & 1.69 & $0.39-8.36$ & 0.4844 \\
Multiple tumors & 4.72 & $1.07-23.4$ & 0.0410 \\
Perihilar tumor & 7.02 & $1.01-147$ & 0.0488 \\
CEA level $>2.2(\mathrm{ng} / \mathrm{ml})$ & 4.12 & $1.00-20.2$ & 0.0496 \\
\hline
\end{tabular}

CEA: Carcinoembryonic antigen. 
Table VI. Recurrence sites after RO resection for intrahepatic cholangiocarcinoma in patients with and without lymph node (LN) involvement

\begin{tabular}{lccc}
\hline Recurrence & With LN involvement $(\mathrm{n}=46), \mathrm{n}$ & Without LN involvement $(\mathrm{n}=13), \mathrm{n}$ & $p$-Value \\
\hline Single liver metastasis & 10 & 0 & 0.02 \\
Multiple liver metastasis & 7 & 4 & 0.23 \\
Lymph node metastasis & 6 & 6 & 0.07 \\
Peritoneal dissemination & 0 & 4 & 0.002 \\
Lung metastasis & 5 & 0 & 0.33 \\
Bone metastasis & 0 & 1 & 0.73 \\
\hline
\end{tabular}

along portal triads, there are also several lymphatic outflows that communicate directly with distant areas, or the general lymphatic system $(15,16)$. Moreover, it has been revealed that ICC tumor spreads to more distal nodes through the hepatoduodenal ligament or other multidirectional lymphatic pathways connected to the general lymphatic system $(15,16)$. Therefore, systematic lymph node dissection might effectively represent only lymph node sampling when there is more extensive occult lymph node metastasis. This underlines the importance of highlighting the risk factors for poor prognosis in patients with ICC, especially those undergoing curative resection. It is still difficult to evaluate lymph node metastasis preoperatively in patients with ICC by computed tomography or positron-emission tomography (17), but it may be possible to predict pathological lymph node involvement by evaluating preoperative tumor characteristics.

In our study, multiple tumors, perihilar tumor location and CEA concentration $>2.2 \mathrm{mAU} / \mathrm{l}$ were independent preoperative predictors of lymph node involvement after R0 resection. Preoperatively, patients with ICC who have one or more of these three factors may benefit from additional preoperative therapy, such as neoadjuvant chemotherapy. In our opinion, pathological lymph node involvement should be considered systemic disease. Katayose et al. have reported the results of their phase I study of neoadjuvant chemoradiation in patients with ICC (5), but further studies are needed to evaluate the therapeutic benefits in patients undergoing curative resection. Patients with ICC and pathological lymph node involvement may also benefit from postoperative adjuvant chemotherapy. Effective surgical management and neo- and adjuvant chemotherapy may be required to prolong patient survival. However, there is no consensus for pre- and postoperative adjuvant therapy in ICC. We currently use a combination of gemcitabine and cisplatin as first-line adjuvant chemotherapy (18). More precise and well-planned analysis of neo- and adjuvant chemotherapy is, therefore, needed. Molecular targeted therapy may also be effective, as it is in other cancers of the digestive tract.

Our study had certain limitations. The sample was relatively small, which made it difficult to evaluate the outcomes of curative resection; however, few previous studies have examined the role of pre- and postoperative treatment of ICC after curative resection. We found a significant difference in OS between the R0N1 and R1 groups. Because the UICC stages in patients with R0N1 disease were less favorable than those with $\mathrm{R} 1$ disease, the OS of patients with recurrence of R0N1 ICC was significantly worse than that of those with R1 disease, even though there was no difference in RFS between the groups.

In conclusion, we found that lymph node involvement was an independent predictor of poorer long-term survival in patients undergoing curative resection for ICC. Those patients with ICC with multiple tumors, perihilar tumors and serum CEA concentration $>2.2 \mathrm{ng} / \mathrm{ml}$ in association with lymph node involvement may need additional preoperative chemotherapy.

\section{Acknowledgements}

The Authors thank Dr. S Aishima from the Department of Pathology and Microbiology, Saga University for diagnosing the pathologic characteristics of ICC.

\section{Conflicts of Interest}

The Authors declare that they have no conflict of interest in regard to this study.

\section{References}

1 Ikai I1, Itai Y, Okita K, Omata M, Kojiro M, Kobayashi K, Nakanuma Y, Futagawa S, Makuuchi M and Yamaoka Y: Report of the 15th follow-up survey of primary liver cancer. Hepatol Res 28: 21-29, 2004.

2 Endo I, Gonen M, Yopp AC, Dalal KM, Zhou Q, Klimstra D, D'Angelica M, DeMatteo RP, Fong Y, Schwartz L, Kemeny N, O'Reilly E, Abou-Alfa GK and Shimada H, Blumgart LH and Jarnagin WR: Intrahepatic cholangiocarcinoma: rising frequency, improved survival, and determinants of outcome after resection. Ann Surg 248: 84-96, 2008.

3 Aljiffry M1, Abdulelah A, Walsh M, Peltekian K, Alwayn I and Molinari M: Evidence-based approach to cholangiocarcinoma: a systematic review of the current literature. J Am Coll Surg 208: 134-147, 2009. 
4 Yamashita Y, Taketomi A, Morita K, Fukuhara T, Ueda S, Sanefuji K, Iguchi T, Kayashima H, Sugimachi K and Maehara Y: The impact of surgical treatment and poor prognostic factors for patients with intrahepatic cholangiocarcinoma: retrospective analysis of 60 patients. Anticancer Res 28: 2353-2359, 2008.

5 Katayose Y, Rikiyama T, Motoi F, Yamamoto K, Yoshida H, Morikawa T, Hayashi H, Kanno A, Hirota M, Satoh K, Ariga H, Suzuki M, Ohyauchi M, Kondo Y, Ikeya S, Ogawa Y, Shimosegawa T, Egawa $\mathrm{S}$ and Unno M: Phase I trial of neoadjuvant chemoradiation with gemcitabine and surgical resection for cholangiocarcinoma patients (NACRAC study). Hepatogastroenterology 58: 1866-1872, 2011.

6 McNamara MG, Walter T, Horgan AM, Amir E, Cleary S, McKeever EL, Min T, Wallace E, Hedley D, Krzyzanowska M, Moore M, Gallinger S, Greig P, Serra S, Dawson LA and Knox JJ: Outcome of adjuvant therapy in biliary tract cancers. Am J Clin Oncol 38: 382-387, 2015.

7 Murakami Y, Uemura K, Sudo T, Hashimoto Y, Nakashima A, Kondo N, Sakabe R, Ohge H and Sueda T: Prognostic factors after surgical resection for intrahepatic, hilar, and distal cholangiocarcinoma. Ann Surg Oncol 18: 651-658, 2011.

8 Sobin LH, Gospodarowicz MK, Wittekind C (eds): International Union Against Cancer (UICC). TMN Classification of Malignant Tumors. Seventh Edition. New York: Wiley-Blackwell, 2010.

9 Yamashita YI, Wang H, Kurihara T, Tsujita E, Nishie A, Imai K, Hashimoto D, Chikamoto A, Aishima S and Baba H: Clinical significances of preoperative classification of intrahepatic cholangiocarcinoma: different characteristics of perihilar $v s$ peripheral ICC: Anticancer Res 36: 6563-6569, 2016.

10 Shimada M, Yamashita Y, Aishima S, Shirabe K, Takenaka K and Sugimachi K: Value of lymph node dissection during resection of intrahepatic cholangiocarcinoma. Br J Surg 88: 1463-1466, 2001

11 Uchiyama K, Yamamoto M, Yamaue H, Ariizumi S, Aoki T, Kokudo N, Ebata T, Nagino M, Ohtsuka M, Miyazaki M, Tanaka E, Kondo S, Uenishi T, Kubo S, Yoshida H, Unno M, Imura S, Shimada M, Ueno M and Takada T: Impact of nodal involvement on surgical outcomes of intrahepatic cholangiocarcinoma: a multicenter analysis by the Study Group for Hepatic Surgery of the Japanese Society of Hepato-Biliary-Pancreatic Surgery. J Hepatobiliary Pancreat Sci 18: 443-452, 2011
12 Dindo D, Demartines $\mathrm{N}$ and Clavien PA: Classification of surgical complications: a new proposal with evaluation in a cohort of 6336 patients and results of a survey. Ann Surg 240: 205-213, 2004

13 Spolverato G, Vitale A, Cucchetti A, Popescu I, Marques HP, Aldrighetti L, Gamblin TC, Maithel SK, Sandroussi C, Bauer TW, Shen F, Poultsides GA, Marsh JW and Pawlik TM: Can hepatic resection provide a long-term cure for patients with intrahepatic cholangiocarcinoma? Cancer 121: 3998-4006, 2015.

14 Doussot A, Groot-Koerkamp B and Wiggers JK, Chou J, Gonen M, DeMatteo RP, Allen PJ, Kingham TP, D'Angelica MI and Jarnagin WR: Outcomes after resection of intrahepatic cholangiocarcinoma: external validation and comparison of prognostic models. J Am Coll Surg 22: 452-461, 2015.

15 Shirabe K, Mano Y, Taketomi A, Soejima Y, Uchiyama H, Aishima S, Kayashima $H$, Ninomiya $M$ and Maehara $Y$ : Clinicopathological prognostic factors after hepatectomy for patients with mass-forming type intrahepatic cholangiocarcinoma: relevance of the lymphatic invasion index. Ann Surg Oncol 17: 1816-1822, 2010

16 Pupulim LF, Vilgrain V, Ronot M, Becker CD, Breguet R and Terraz S: Hepatic lymphatics: anatomy and related diseases. Abdom Imaging 40(6): 1997-2011, 2015.

17 Morine Y and Shimada M: The value of systemic lymph node dissection for intrahepatic cholangiocarcinoma from the viewpoint of liver lymphatics. J Gastroenterol 50: 913-927, 2015.

18 Adachi T, Eguchi S, Beppu T, Ueno S, Shiraishi M, Okuda K, Yamashita Y, Kondo K, Nanashima A, Ohta M, Takami Y, Noritomi T, Kitahara $\mathrm{K}$ and Fujioka H: Prognostic impact of preoperative lymph node enlargement in intrahepatic cholangiocarcinoma: a multi-institutional study by the Kyushu Study Group of Liver Surgery. Ann Surg Oncol 22: 2269-2278, 2015.

Received March 14, 2017

Revised May 17, 2017

Accepted May 19, 2017 